\title{
Tissue engineering through mesenchymal stem cells: role of the $G$ enostem Consortium
}

with expertise in complementary fields. Within the consortium, teams with a focus on MSC biology and characterization, and the study and identification of growth factors, work in close co-operation with specialists in biomaterials, bioinformatics, genomics and molecular biology. Teams with expertise in animal models for bone, tendon and cartilage defects are collaborating to identify the optimal preclinical applications. The biomechanical evaluation of the results obtained forms the final part of the project. Thus, the G enostem teams aim to characterize a signature of human and murine undifferentiated M SC s at the genomic and proteomic level. The identification of the differentiation step to obtain mature chondrocytes through BM P2 or TGF $\beta_{3}$ induction has been shown and compared with the gene profile of adipocytes or osteocytes at four time points (between day 1 and day 21). In addition, requirements for standardization of the procedures for the production and expansion of the MSCS has been defined and circulated within the consortium. O nly by developing the competence of cellular production within G enostem to GM P standards, will it be possible to start to consider future therapeutic implications. A better knowledge of the M SC 'niche', including cell adhesion, chemokine expression and cellular interactions with the endothelium of the hematopoïetic cells or the skeletal tissue environment will also contribute to future advances. associated with biomaterials and implanted in affected tissue must be investigated and understood before use in clinical applications such as bone defect, tendon rupture, osteoarthritis $(\mathrm{OA})$ or rheumatoid arthritis (RA).

This overview highlights the range of research devoted to this topic underway within the different centers across Europe that comprise the Genostem European U nion Project.

\section{Genostem Consortium}

Without doubt, the Genostem partners have strongly contributed to a dynamic European research in the field of adult stem cells. Genostem is a project funded within the European Commission's 6th framework program and includes 22 teams from 11 European countries

\section{Challenges of tissue repair}

Tendon rupture, large cartilage defect or bone defect are all difficult to repair and may lead to physical limitation for the patient. Nonunion after bone fracture or bone reconstruction following surgery could be addressed using bone progenitors combined with osteo-inductive scaffolds. 0 steoporosis is common among the elderly, and theincidence of hip fracture among this group has a high socioeconomic impact. In spite of the therapeutic innovations, such as bisphosphonates, strontium ralenate or parathormone treatment, the incidence of the risk of repetition of vertebral fracture after the first instance remains increased. $O A$ of the lower limbs, a cause of significant handicap, affects nearly $16 \%$ of the population 
aged more than 60 years. Cartilage lesions are irre versible, and joint prosthesis is currently the only therapeutic option available for advanced stages of RA or OA. Cell therapy involving transplantation of autologous cells combined with scaffolds able to regenerate the cartilage matrix specifically in the damaged areas therefore opens up new possibilities. RA is a common inflammatory joint pathology that affects $0.3 \%$ of the EU population, and enormous progress has been made obtained thanks to the biotherapies such as T N F- $\alpha$ or IL-1 blocking agents.

M ost of these skeletal pathologies are accompanied by tendon distension or rupture, with associated pain. Animal models demonstrating the regeneration of skeletal tissue through the use of adult stem cells (M SCs) open up exciting therapeutic prospects for such patients. Nevertheless, the development of M SC s as therapeutic tools requires full understanding of the stability of the phenotype obtained and the functionality of the regenerated tissue, with the same safety requirements that would be necessary for the use of any drug.

Key role of mesenchymal stem cells MSC S are pluripotent cells and are present in many adult mesenchymal tissues as synovium, muscle, adipose tissue and BM . M SC s are isolated from $\mathrm{BM}$ and can be expanded ex vivo without loss of phenotype or multipotentiality [3]. M SC S are identified by their phenotype, their triple potentiality and their properties for self renewal. Among other factors, MSCs express Thy-1 (CD 90), endoglin (CD 105), vascular cell adhesion molecule (VCAM )-1 (CD 106) and are negative for the hematopoietic markers (CD 34, CD 45, CD 14) (reviewed in [4]). Recent studies have shown that the cell concentration is a critical parameter for culture [2]. M SC s are also defined initially by their function; they are pluripotent cells and can lead to different lineages including cartilage, adipose tissue, bone or tendon tissue. Finally, these cells are characterized by their capacity for self renewal with asymmetrical division. $N$ evertheless, description of these cells in the literature remains heterogeneous, and there is a clear need for the European research community to define standards for use and optimization of conditions of culture.

Adult stem cells in bone, cartilage \& tendon tissue repair

Several options have been proposed for the use of stem cells in the development of reconstructive therapy. O ne possibility is re-injection of the M SC s at an undifferentiated stage but associated with scaffolds and specific differentiation factor (or a combination of factors). O ne can consider genetic transformation of the cell to express this factor on an autocrine basis or to express an active transcription factor [5]. H owever, to better control the phenotype it would be more judicious to plan ex vivo differentiation of M SC s in order to obtain mature and functional tissue before implantation at the requisite site. This would allow validation of the phenotype, its stability and the homogeneity of the cells injected. Thus, standardization of the conditions of differentiation in the mesenchymal lineage is an essential step before considering the clinical applications. An alternative novel approach involves redifferentiation of the $\mathrm{M} \mathrm{SC}$ s present in the injured tissue. It has been shown that within bone or cartilage tissue, there exists a small number of undifferentiated cells with the markers characteristic of the M SC [6]. Fewer than 5\% of the cells of the cartilage consist of undifferentiated cells, although it is not clear whether these are dedifferentiated chondrocytes, quiescent M SC S or progenitors that have migrated from the BM . N evertheless, there is the possibility that these M SC s could be reactivated for optimal differentiation. H owever, in inflammatory diseases, such as $\mathrm{OA}$ or RA, the environment for differentiation will not be optimal [7]. We have shown that IL- 1 or TN F- $\alpha$ can inhibit differentiation in spite of significant concentrations of BM P2 or of TGF $\beta_{1}$. These inflammatory conditions must be controlled before considering this type of local therapeutic approach.

\section{Potential applications}

There are some potential limitations to the use of M SC s for medical applications deserving consideration. It has been shown that stem cells fuse to endogenous differentiated cells, forming tetraploid cells in vivo, although this fusion phenomenon seems to be extremely rare [8]. Also, M SC s have also been shown to inhibit $\mathrm{T}$-cell proliferation through an immunosuppressive effect mediated by $C D 8$ regulatory $T$ cells [9].

In animal models, M SC s delay graft rejection and permit tumor growth in allogeneic recipients [10]. This raises the question of whether M SC S could favor the emergence of tumors in patients receiving $\mathrm{MSC}$ s for tissue repair. In experimental models of gastric cancer, a progressive migration of MSC $S$ originating from $\mathrm{BM}$ has been shown [11]. These M SC s were labelled 
and were involved in the formation of gastric epithelial tumors. H ence, there is an indication that BM M SC s may support of tumor proliferation or transformation of the cells. Within tumors, cells have been identified with stem cell membrane markers including CD $34^{+} \mathrm{CD} 113^{+}$ but not THY 1, CD 38 or CD 24. The expression of telomerase and the transcription factor BM I1, which represses the cell cycle genes INK 4P16 and P14 in MSCs, brings them closer to the tumor cells [12]. Recent data show that tumor stromal cells drive spontaneous induction of the catenin pathway and TGF- $\beta$ signalling activation, suggesting possible CSM crosstalk within the tumor. However, a recent case study of a patient with severe treatment-resistant G rade IV acute graft versus host disease showed encouraging results of the efficacy of M SCs as immunoregulatory cells, one year after the treatment and without any adverse effect [13].

We, and others, have demonstrated the immunosuppressive properties of MSC in vivo [14]. M SC $s$ were shown to secrete cytokines, in particular IL6. M SCs in coculture also have an inhibitory effect on the maturation of dendritic cells and on NK T-cells [15]. Finally, an enzymatic activity ID 0 which inhibits tryptophan metabolism could explain the antiproliferative effect on T-lymphocytes. IDO activity is induced through IFN - $\gamma$ after cellular interactions [16].

A further limitation may be the need to maintain the differentiated state, and tight control is required to avoid unwanted dysregulation of the process. D evelopment of inducible promoters to regulate the expression of differentiating factors or the use of scaffolds that could progressively deliver an active molecule has to be tested within Genostem.

\section{Summary}

Tissue engineering for cartilage and bone repair is an exciting and realistic goal. M SC s seem to be the best candidates for cell therapy to regenerate injured skeletal tissues owing to the ease of isolation, expansion and multipotentiality. These cells can be induced to differentiate into chondrocytes, tendinocytes or osteoblasts when subjected to specific environmental factors or when expressing specific transcription factors or signalling molecules $[17,18]$. H owever, regeneration of a true functional tissue requires the use of fully characterized MSCs, biodegradable scaffolds and selective differentiating factors, which have yet to be fully identified. Finally, the longterm behavior of M SC s embedded in biomaterials in the context of diseased joints or bone, remains to be established before we can progress to clinical trials.

The priority in developing M SC -based therapy must be the safety of the patient. It is necessary to obtain, before implantation, perfectly homogeneous cells that are well characterized on phenotypic and biological levels. Studies of the absence of carcinogenic transformation are essential, including chromosomal studies, tert expression or in vivo transformation assay. The regulatory agencies will require the immunological characteristics of these cells to be specified before the clinical use. Finally, European health agencies will require the use of medium without serum to obtain better standardization of culture conditions and safety of use. The G enostem Consortium is directing its efforts in this direction, and the time is not far away when we will be ableto propose a clinical program with well characterized cells, homogeneous with intact functional properties with the aim of bone regeneration.
Bibliography

1. Smith JR, Pochampally R, Perry A, H su SC, Prockop DJ: I solation of a highly clonogenic and multipotential subfraction of adult stem cells from bone marrow stroma. Stem Cells. 22, 823-831 (2004).

2. Colter DC, Class R, DiG irolamo CM , Prockop DJ: Rapid expansion of recycling stem cells in cultures of plastic-adherent cells from human bone marrow. Proc. $N$ atl Acad. Sci. U SA 97, 3213-3218 (2000).

3. Pereira RF, $\mathrm{H}$ alford $\mathrm{KW}, \mathrm{O}^{\prime} \mathrm{H}$ ara $\mathrm{MD}$ et al.: Cultured adherent cells from marrow can serve as long-lasting precursor cells for bone, cartilage, and lung in irradiated mice. Proc. Natl Acad. Sci. U SA 92, 4857-4861 (1995).

4. N oël D, D jouad F, Jorgensen C: Regenerative medicine through mesenchymal stem cells for bone and cartilage repair. Curr. 0 pin. Investig. D rugs 3 , 1000-1004 (2002).

5. Bi W, D eng J M , Zhang Z, Behringer RR, de Crombrugghe $B$ : Sox9 is required for cartilage formation. $\mathrm{N}$ at. $\mathrm{G}$ enet. $22,85-89$ (1999).

6. Alsalameh S, Amin R, G emba T, Lotz M . Identification of mesenchymal progenitor cells in normal and osteoarthritic human articular cartilage. Arthritis Rheum. 50, 1522-1532 (2004).

7. Koshy PJ, H enderson N, Logan C, Life PF, Cawston TE, Rowan AD : interleukin-17 induces cartilage collagen breakdown: novel synergistic effects in combination with proinflammatory cytokines. Ann. Rheum. Dis. 2002 61:704-13.

8. Spees JL, O Ison SD, Y Yostalo Y et al.: Differentiation, cell fusion, and nuclear fusion during ex vivo repair of epithelium by human adult stem cells from bone marrow stroma. Proc. Natl Acad. Sci. U SA 100, 2397-2402 (2003). 
9. D jouad F, Plence $P$, Bony $C$ et al.: Immunosuppressive effect of mesenchymal stem cells favors tumor growth in allogeneic animals. Blood 102, 3837-3844 (2003).

10. Bartholomew A, Sturgeon C, Siatskas M et al.: M esenchymal stem cells suppress lymphocyte proliferation in vitro and prolong skin graft survival in vivo. Exp. H ematol. 30, 42-48 (2002).

11. H oughton J, Stoicov C, N omura S et al.: $\mathrm{G}$ astric cancer originating from bone marrow-derived cells. Science 306, 1568-1571 (2004).

12. Peters BA, D iaz LA, Polyak $K$ et al.: Contribution of bone marrow-derived endothelial cells to human tumor vasculature. $N$ at. M ed. 11, 261-262 (2005).
13. LeBlanc K, Rasmusson I, Sundberg B et al.: Treatment of severe acute graft versus host disease with third party haploidentical mesenchymal stem cells. Lancet 363, 1439-1441 (2004).

14. Le Blanc K, Tammik L, Sundberg B, $H$ aynesworth $S E$, Ringden $O$ : M esenchymal stem cells inhibit and stimulate mixed lymphocyte cultures and mitogenic responses independently of the major histocompatibility complex. Scand. J. Immunol. 57, 11-20 (2003).

15. D jouad F, Fritz V, Apparailly $F$ et al.: Reversal of the immunosuppressive properties of mesenchymal stem cells by tumor necrosis factor al pha in collagen- induced arthritis. Arthritis Rheum. 52(5),1595-1603 (2005).

16. Krampera M, Cosmi L, Angeli R et al.: Role for Interferon-gamma in the Immunomodulatory Activity of $\mathrm{H}$ uman Bone $M$ arrow M esenchymal Stem Cells. Stem Cells 24, 386-398 (2006).

17. Jorgensen C, Gordeladze J, N oel D: Tissue engineering through autologous mesenchymal stem cells. Curr. 0 pin. Biotechnol. 15(5), 406-410 (2004).

18. Gazit $D$, Turgeman $G$, Kelley $P$ et al.: Engineered pluripotent mesenchymal cells integrate and differentiate in regenerating bone: a novel cell-mediated gene therapy. J. Gene M ed. 1, 121-133 (1999). 